\title{
Radial Head Fractures: Mason Johnston's Classification Reproducibility
}

\author{
RES Pires*, MPH, FL Rezende*, MD, EC Mendes, MD, AER Carvalho Jr*, MD, IA Almeida Filho*, MD, \\ FB Reis**, PhD, MAP Andrade***, PhD
}

\author{
*Department of Orthopaedic Surgery, Felicio Rocho Hospital, Belo Horizonte, Brazil \\ **Department of Orthopaedic Surgery, Federal University of Sao Paulo, Sao Paulo, Brazil \\ ***Department of Orthopaedic Surgery, Federal University of Minas Gerais, Belo Horizonte, Brazil
}

\begin{abstract}
Background: The purpose of the study was to evaluate interand intra-observer reproducibility of Mason Johnston classification of radial head fractures in adults. Materials and Methods: Thirty radiographic images of radial head fractures (anteroposterior and lateral views) from adult patients were examined by five professionals in the field of Orthopaedics and Traumatology, including an expert member of the Brazilian Society of Orthopaedics, an experienced orthopaedic surgeon specialising in the elbow and shoulder, and three resident physicians (one each in the 1st, 2nd and 3rd years of residency at an Orthopaedics and Trauma unit). Inter- and intra-observer concordances were

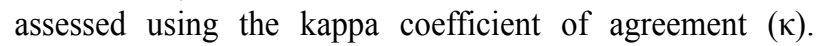
Results: The intra-observer reproducibility of the Mason Johnston classification system ranged from moderate to good whilst the inter-observer agreement varied from weak to good. Conclusion: The Mason Johnston's classification system, although used throughout the world, possesses questionable reproducibility with respect to the assessment of radial head fractures. The level of professional expertise did not influence the outcome of the study.
\end{abstract}

Key Words:

Radial Head Fractures, Classification Systems, Reproducibility, Mason Johnston's Classification System

\section{INTRODUCTION}

The intra-articular position of the radial head stabilised the elbow and forearm and enables full range of elbow motion. Fractures of the radial head are involved in approximately $20 \%$ of elbow trauma cases and between $5-10 \%$ of elbow dislocations are associated with fractures of the radial head, typically caused by falling onto an outstretched wrist, the elbow semi-flexed and the forearm in full pronation'. The male to female ratio in these injuries is $1: 2$ and approximately $80 \%$ of occur in adults (mean age: 30 years). Appropriate treatment depends on fracture type, associated soft tissue lesions and functional demands of the patient. It is important to develop a pre-surgical plan that includes correct classification of the fracture type in order to establish the correct treatment. Such a classification must be simple and reproducible, should indicate the prognosis and act as a treatment guide ${ }^{2}$. The literature contains previous evaluations of the reproducibility of several classification systems for radial head fractures, including Hotchkiss, AO, Mason (without the modification proposed by Johnston), Morrey and Mayo ${ }^{3,4,5}$.

Mason, in 1954, described his classification system, which includes three fracture types defined as: type I, undisplaced fractures; type II, displaced, involving more than $30 \%$ of the radial head but less than 50\%; type III, comminuted fracture involving the entire head. Johnston (Figure 1), in 1962, added a fourth type (radial head fracture and ulnohumeral dislocation) to the Mason classification. The MasonJohnston classification of these four fracture types persists as the main classification system for fractures of the radial head and is the most frequently used system in English medical literature. The aim of the present study was to evaluate interand intra-observer reproducibility of the Mason Johnston classification of radial head fractures.

\section{MATERIALS AND METHODS}

The study was approved by the institutional Ethical Committee and performed according to the Declaration of Helsinki standards. Anteroposterior and lateral radiographs of thirty radial head fractures in adult patients were randomly selected from the hospital archives and converted into digital images using a 3.2 megapixel camera. The images associated with detailed explanations regarding Mason Johnston's classification system, were stored on a CD-ROM (Figure 1). The radiographic images were then analysed by five different professionals in the field of Orthopaedics and Traumatology, including an expert member of the Brazilian Society of Orthopaedics and Traumatology (SBOT expert), a senior shoulder and elbow surgeon, and three orthopaedic residents during their 1st, 2nd and 3rd year of residency at the Orthopaedics and Trauma unit. None of the observers 
Radial Head Fractures: Mason Johnston's Classification Reproducibility

Table I: Kappa coefficients $(\kappa)$ of the intra-observer agreement relating to the classification of radial head fractures by the Mason Johnston system

\begin{tabular}{|lccc|}
\hline Observer & Coefficient of concordance (к) & 95\% Confidence interval & $\rho$ values \\
\hline 1st Year resident physician & 0.520 & $0.2659-0.7741$ & 0,0000 \\
2nd Year resident physician & 0.513 & $0.2683-0.7577$ & 0,0000 \\
3rd Year resident physician & 0.650 & $0.4205-0.8795$ & 0,0000 \\
SBOT expert & 0.7685 & $0.5859-0.9511$ & 0,0000 \\
Orthopaedic surgeon & 0.5631 & $0.3209-0.8053$ & 0,0000 \\
\hline
\end{tabular}

Table II: Kappa coefficients ( $\mathrm{k}$ ) of the inter-observer agreement relating to the classification of radial head fractures by the Mason Johnston system

\begin{tabular}{|c|c|c|}
\hline Observers & $\mathrm{K}$ at first evaluation & $\mathrm{K}$ at second evaluation \\
\hline SBOT expert $\times$ orthopaedic surgeon & 0.400 & 0.676 \\
\hline SBOT expert $\times$ 3rd year resident & 0.491 & 0.579 \\
\hline SBOT expert $\times 2$ nd year resident & 0.399 & 0.720 \\
\hline SBOT expert $\times 1$ st year resident & 0.537 & 0.486 \\
\hline Orthopaedic surgeon $\times 3$ rd year resident & 0.507 & 0.453 \\
\hline Orthopaedic surgeon $\times 2$ nd year resident & 0.562 & 0.662 \\
\hline Orthopaedic surgeon $\times 1$ st year resident & 0.562 & 0.475 \\
\hline $3 r d \times 2$ nd year resident & 0.416 & 0.556 \\
\hline $3 r d \times 1$ st year resident & 0.660 & 0.370 \\
\hline $2 \mathrm{nd} \times 1$ st year resident & 0.557 & 0.665 \\
\hline
\end{tabular}

Underlined values represent the highest and lowest coefficients
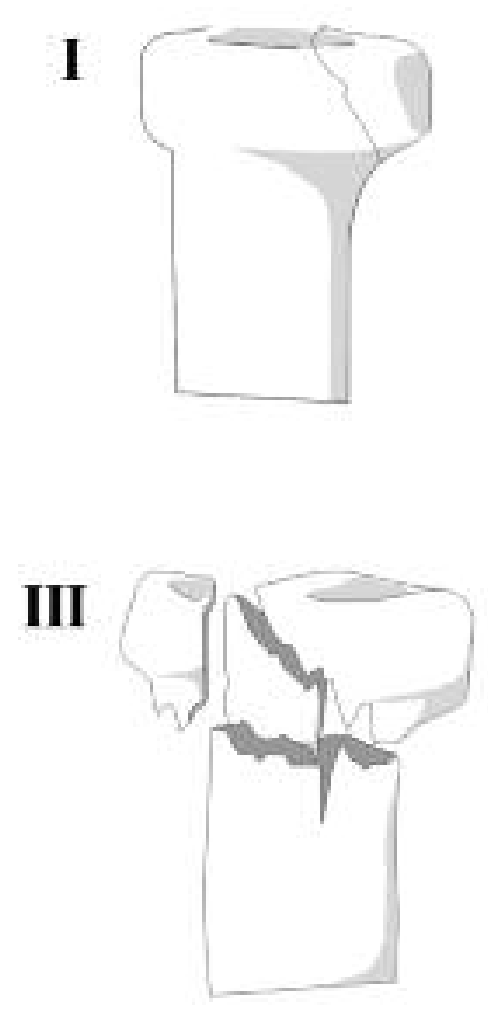

Fig. 1: Mason Johnston's Classification (Types I-IV).
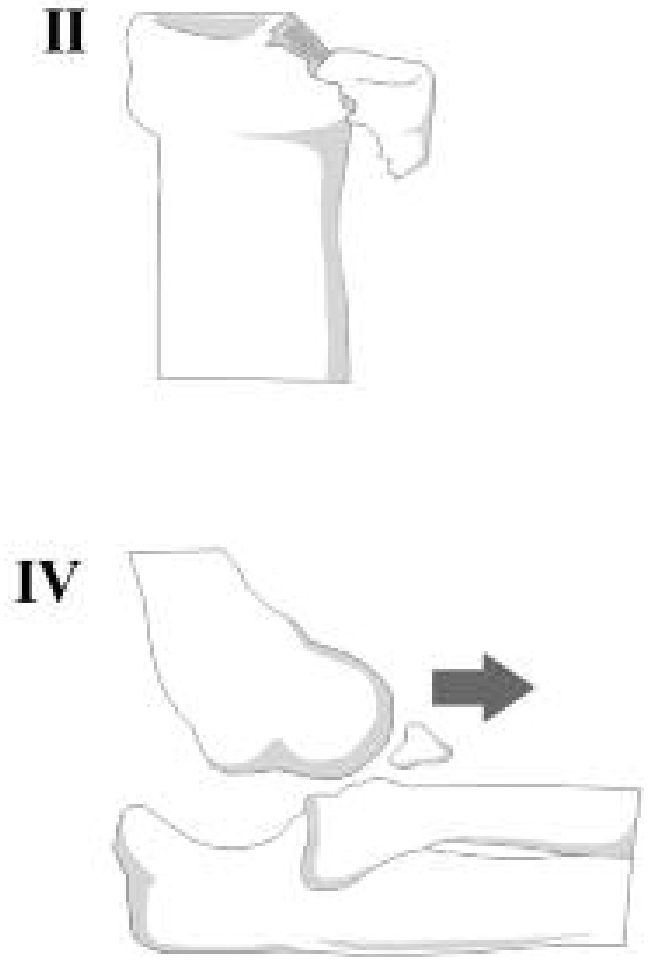
had previous knowledge of the cases or were involved in the management of the patients included in the study. Observers were advised to consider radial head displacement using the criteria of $2 \mathrm{~mm}$. Fractures were classified based on direct visual analysis of the radiographs. There was no specific period of time allotted for radiographic analyses. Inter- and intra-observer reproducibility was measured by conducting a second evaluation of the same images, presented in a different order, after a minimal interval of 30 days. As the classification was based only on x-ray observation, soft tissue lesions or signs of instability were not taken into consideration.

Sample size was based on standard statistical parameters and on previous studies. The inter and intra-observer degrees of concordance were assessed from the kappa coefficient of agreement $(\kappa)$, stratified according to Landis and Koch ${ }^{6}$, as poor $(\kappa<0.02)$, weak $(0.21 \geq \kappa \leq 0.40)$, moderate $(0.41 \geq \kappa$ $\leq 0.60)$, good $(0.61 \geq \kappa \leq 0.80)$ and very good $(\kappa>0.80)$. Kappa is a measure that describes the correlation between two "judges" or one "judge" in two separate stages. It is used in situations containing categorical variables (not numeric). Some authors have used the Mann-Whitney Test as a method to evaluate reproducibility of classification systems, as it is used to compare two independent samples (i.e., it is similar to a nonparametric Student $t$ Test). The Mann-Whitney test is used when the investigator wants to know whether there is a significant difference between two independent samples, and when there exist quantitative variables (numerical data). Therefore, the Mann-Whitney test was not appropriate for use in this study. When using the kappa coefficient, there are no restrictions on sample size. The level of statistical significance for the present study was established at $p \leq 0.05$. This method of statistical analysis has been used as in the great majority of similar papers published in the literature.

\section{RESULTS}

The quality of the digital radiographic images was considered satisfactory for the purpose of classifying these radial head fractures. Inter- and intra-observer reproducibility values were lower than 0.002, indicating significant statistically association for both parameters. Intra-observer concordances varied from moderate to good (p: 0.0000), as shown in Table I. Intra-observer concordance levels among the highly experienced professionals was not better than those attained by the less experienced group of resident physicians, although the highest degree of intra-observer agreement was registered by the SBOT expert $(\kappa=0.7685)$. As shown in Table II, the degree of concordance between the senior professionals (SBOT expert, and senior orthopaedic surgeon) was low at the first evaluation $(\kappa=0.400)$, but increased at the second evaluation $(\kappa=0.676)$. The highest concordance was observed between the SBOT expert and the 2nd year resident physician $(\kappa=0.720)$, whereas the lowest was between 3rd and 1st year resident physicians $(\kappa=0.370)$, both at the second evaluation.

\section{DISCUSSION}

The radial head transmits power across the whole arm and lends valgus stability to the elbow and its medial collateral ligament. Radial head fractures, which are mainly caused by falls onto an extended wrist with the forearm slightly flexed and in pronation, may be associated with elbow dislocation and ulna fracture of the coronoid process. Patients presenting with this type of injury complain of pain during pronosupination of the forearm. Such fractures comprise approximately $20 \%$ of elbow traumas ${ }^{3}$. Although anteroposterior and lateral radiographs are enough for accurate diagnosis, oblique views can be useful in certain circumstances ${ }^{1}$.

The Mason Johnston classification is used in the majority of orthopaedic and trauma services worldwide in order to establish the diagnosis and treatment of radial head fractures. It is also used for prognosis and identification of soft tissues injuries. The classification of radial head fractures attempts to distinguish between fractures that may be treated by cast, fractures that are amenable to internal fixation, and fractures that cannot be reconstructed and instead requires prosthetic replacement or excision ${ }^{4}$. However, classification systems must be reproducible, easily understood and memorized, concise, practical and provide treatment guidelines and prognosis.

Some studies have focussed on the inter- and intra-observer concordance of various classification systems relating to radial head fractures ${ }^{7}$. Sheps et $a l^{4}$, assessed the reproducibility of classification systems for radial head fractures (AO Classification and the Hotchkiss modification of the Mason Classification) and concluded that the AO classification was fair according to the criteria of Landis and Koch. The Hotchkiss classification has a moderate interobserver reliability, with a Kappa of 0.585 . Intra-observer agreement for the Morrey classification was excellent, while inter-observer agreement was moderate ${ }^{8,9}$. The Mayo Classification includes associated ligaments and osseous injuries of the elbow and shows fair to moderate interobserver agreement, while intra-observer agreement is fair ${ }^{10}$.

Morgan et $a^{11}$, analysing the Mason Classification (without the Johnston modification), found poor intra-observer reproducibility, and a kappa index of 0.45 for the interobserver assessment, demonstrating moderate reproducibility. Dillon et $a^{12}$, also found fair to moderate inter-observer reproducibility when evaluating the reproducibility of the Mason Classification (without the Johnston modification) for fractures of the radial head. They emphasized that the oblique radiograph with external rotation had improved inter-observer reproducibility and can be helpful in preoperative planning. 
The present study shows that inter-observer reproducibility of the Mason Johnston's system ranged from weak to good, whereas intra-observer reproducibility ranged from moderate to good. It seems, however, that the reproducibility associated with the classification is not influenced by the degree of professional expertise of the observer, since the inter-observer concordances between senior experts (SBOT expert and orthopaedic surgeon) were as unsatisfactory as those obtained when the observers were junior professionals (resident physicians) were analysed.

\section{CONCLUSION}

Investigation of the efficacy of Mason Johnston classification system in assessment and comparison of radial head fractures showed questionable reproducibility results for radial head fractures. The degree of professional expertise did not influence the outcome of the study. 


\section{REFERENCES}

1. Morrey BF, An KN, Stormont TJ. Force transmission through the radial head. J Bone Joint Surg 1988; 70A: 250-6.

2. Johnston GW. A follow-up of one hundred cases of fractures of the head of the radius with a review of the literature. Ulster Med $J$ 1962; 31: 51-6.

3. Morrey BF, Tanaka S, Na KN. Valgus stability of the elbow: a definition of primary and secondary constraints. Clin Orthop Relat Res 1991; 265: 187-95.

4. Sheps DM, Kiefer KRL, Boorman RS, DonaghyJ, Lalani A, Walker R, et al. The interobserver reliability of classification systems for radial head fractures: the Hotchkiss modification of the Mason classification and the AO classification systems. Can J Surg 2009; 52(4): 277-82.

5. Matsunaga FT, Tamaoki MJS, Cordeiro EF, Uehara A, Ikawa MH, Matsumoto M, et al. Are classifications of proximal radius fractures reproducible?. BMC Musculoskelet Disord 2009; 10: 1-5.

6. Landis JR, Koch GC. The measurement of observer agreement for categorical data. Biometrics 1977; 33: 159-74.

7. Kaas L, Jupiter JB, van Dijk CN, Eygendaal D. Management of radial head fractures: current concepts. J Shoulder Elbow Surg 2011; 3(1): 34-40.

8. Broberg MA, Morrey BF. Results of treatment of fracture dislocations of the elbow. Clin Orthop Relat Res 1987; 216: 109-19.

9. Doornberg J, Elsner A, Kloen P, van Dijk CN, Ring D. Apparently isolated partial articular fractures of the radial head: prevalence and reliability of radiographically diagnosed displacement. J Shoulder Elbow Surg 2007; 16: 603-8.

10. van Riet RP, Morrey BF. Documentation of associated injuries occurring with radial head fracture. Clin Orthop Relat Res 2008; 466: $130-4$.

11. Morgan SJ, Itamura JM, Shankwiller J, Brien WW, Kuschner SH. Reliability evaluation of classifying radial head fractures by the system of Mason. Bull Hosp Jt Dis 1997; 56(2): 95-8.

12. Dillon MT, Getz CL, Beredjiklian PK, Wiesel BB, Carolan GF, Ramsey M. Evaluation of reliability of the Mason Classification for radial head fractures. Am J Orthop 2010; 39(9): 430-2. 